\title{
Square-wave voltammetry of 5-fluorouracil
}

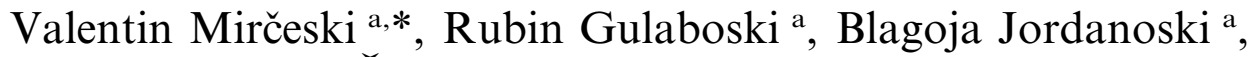 \\ Šebojka Komorsky-Lovrić ${ }^{\mathrm{b}}$ \\ a Institute of Chemistry, Faculty of Natural Sciences and Mathematics, 'Sv. Kiril i Metodij' University, PO Box 162, \\ MK-91001 Skopje, Macedonia \\ ${ }^{\mathrm{b}}$ Center for Marine Research Zagreb, 'Ruđ̈er Bošković' Institute, PO Box 180, 10002 Zagreb, Croatia
}

Received 7 September 1999; received in revised form 26 April 2000; accepted 16 June 2000

\begin{abstract}
The redox reaction of 5-fluorouracil (FU) at a hanging mercury drop electrode (HMDE) is studied by means of square-wave voltammetry (SWV). It is demonstrated that the redox reaction proceeds according to the $\operatorname{scheme:~} \mathrm{L}^{2-}(\mathrm{aq}) \leftrightharpoons \mathrm{L}^{2-}(\mathrm{ads})+$ $\mathrm{Hg}(\mathrm{l}) \leftrightharpoons \mathrm{HgL}(\mathrm{s})+2 \mathrm{e}^{-}$, which involves both chemisorption of $\mathrm{FU}$ on the electrode surface and creation of a sparingly soluble compound with the electrode material. The overall response exhibits properties of a surface process in which both the reactant and the product of the redox reaction are immobilized on the electrode surface. The square-wave voltammetric response of FU possesses features typical of surface confined processes such as 'split SW peaks' and a 'quasi-reversible maximum'. The proposed electrode mechanism is studied theoretically. The numerically calculated response under conditions of SWV is in qualitative agreement with the experimental data. Comparing the theoretical and the experimental data, the kinetic parameters of the redox reaction investigated are estimated. The standard rate constant appears to be within the interval $54 \leq k_{\mathrm{s}} / \mathrm{s}^{-1} \leq 108$, the adsorption constant is $K=10 \mathrm{~cm}^{-1}$, and the transfer coefficient is $\alpha=0.54 \pm 0.01$. The effect of the Cu(II) ions on the adsorptive SWV response of FU is discussed from an analytical point of view. It is demonstrated that SWV is a particularly appealing technique, which enables determination of FU at an ultra-trace concentration level. A linear calibration plot was established at $10^{-11}$ mol $1^{-1}$ concentration level with a correlation coefficient of $R^{2}=0.992$. The detection limit is $7.7 \times 10^{-12}$ mol $1^{-1}$. The reproducibility of the results in terms of the relative standard deviation ranges from 0.9 to $3.2 \%$. C 2000 Published by Elsevier Science S.A.
\end{abstract}

Keywords: Square-wave voltammetry; 5-Fluorouracil; Quasi-reversible maximum

\section{Introduction}

Purine and pyrimidine bases, nucleosides and nucleotides are essential constituents of nucleic acids and enzyme cofactors required for the proper functioning of cells, tissues and organs. A variety of uracil derivatives have been reported as anti-tumor or anti-viral agents or both. Among them, 5-fluorouracil (FU) is particularly important. As an anti-neoplastic agent, it has been used for the treatment of solid tumors of the breast and rectum [1]. Several methods for the determination of organic bases including FU and its metabolites by high-performance liquid chromatography (HPLC) with ultraviolet detection have been described [2,3]. Polaro-

\footnotetext{
* Corresponding author. Fax: + 389-91-226865.

E-mail address: valentin@iunona.pmf.ukim.edu.mk (V. Mirčeski).
}

graphic properties of a series of uracil derivatives have been described for the first time by Manousek and Zuman [4]. These investigators observed anodic polarographic waves of uracils, which appeared to be due to their ability to create sparingly soluble compounds with a mercury electrode. Wrona and Czochralska [5] reported a four electron reduction process of FU at rather negative potentials. Utilizing the capability of FU to create slightly soluble mercury salts, several cathodic stripping methods for its quantitative determination have been developed [6-9].

A detailed study of 18 uracil derivatives, including FU, by dc polarography, differential pulse polarography, and differential pulse cathodic stripping voltammetry has been performed by Bouzid and Macdonald [6]. These authors concluded that two electrons are involved in the redox reaction of mercury in the pres- 
ence of FU due to formation of sparingly soluble complexes. Using differential pulse cathodic stripping voltammetry, a linear calibration plot was established within the concentration range from 0.5 to $5 \times 10^{-7}$ mol $1^{-1}$. Bouzid and Macdonald found that the cathodic stripping response of FU is markedly sensitive to the presence of $\mathrm{Cu}(\mathrm{II})$ ions [6]. Recently, Khodari at al. [9] developed a cathodic stripping voltammetric method for quantitative determination of $\mathrm{FU}$ in the presence of $\mathrm{Cu}$ (II) ions using linear sweep voltammetry. A certain amount of $\mathrm{Cu}(\mathrm{II})$ ions enhance the sensitivity of the cathodic stripping response, lowering the detection limit of the method down to $4.6 \times 10^{-10} \mathrm{~mol} \mathrm{1}^{-1}$. However, the cathodic stripping mechanism in the presence of $\mathrm{Cu}$ (II) ions is still not established.

Guerrier at al. [10] studied the anodic behavior of the mercury electrode in the presence of FU using different electrochemical techniques. The redox reaction was characterized as a process in which both reactant and product are adsorbed on the electrode surface. If the coverage of the electrode increases, a transition between two distinct mechanisms involving the surface products of different stoichiometry was observed.

In this paper, the adsorptive stripping voltammetric behavior of FU by cyclic staircase (CSV) as well as square-wave voltammetry (SWV) is investigated, the objective being to clarify the complex mechanism of this important compound at a hanging mercury drop electrode (HMDE). The aim of the work is also to estimate the redox kinetic parameters, as well as the adsorption constant of FU on the HMDE, since the sensitivity of this particular adsorptive stripping method depends mainly on these parameters. For this purpose, a theoretical model of an electrode mechanism corresponding to the redox reaction of $\mathrm{FU}$ is developed. Moreover, it is demonstrated that SWV, prior to its analytical application, can serve as a powerful technique for total characterization of complex redox processes involving both creation of an insoluble salt with the electrode material and adsorption of the electroactive species. By extension, the theory presented can serve for elucidation and better understanding of the electrode mechanisms of other uracil derivatives, which are known to exhibit similar voltammetric behavior.

In analytical methods for determination of FU reported so far [6-9], it is pointed out that the sensitivity of a particular method can be significantly enhanced in the presence of $\mathrm{Cu}(\mathrm{II})$ ions. The theoretical results presented in this paper provide a base for understanding the influence of $\mathrm{Cu}(\mathrm{II})$ on the voltammetric response of FU.

In the presence of $\mathrm{Cu}(\mathrm{II})$ ions $\mathrm{SWV}$ exhibits the highest sensitivity to the concentration of FU, providing its quantitative determination at ultra-trace concentration level.

\section{Experimental}

All chemicals used were of analytical reagent grade. FU is a product of Henkel (Germany), while $\mathrm{Na}_{2} \mathrm{SO}_{4}$, $\mathrm{H}_{3} \mathrm{PO}_{4}, \mathrm{H}_{3} \mathrm{BO}_{3}, \mathrm{CH}_{3} \mathrm{COOH}$, and $\mathrm{NaOH}$ were from Merck. Britton-Robinson buffers $\left(0.11^{-3}\right)$ prepared as a mixture of $\mathrm{H}_{3} \mathrm{PO}_{4}, \mathrm{H}_{3} \mathrm{BO}_{3}, \mathrm{CH}_{3} \mathrm{COOH}$, and $\mathrm{NaOH}$, were used for adjusting the $\mathrm{pH}$ of the medium. All kinetic measurements were performed in $1 \mathrm{~mol}^{-1}$ $\mathrm{Na}_{2} \mathrm{SO}_{4}$ with $\mathrm{pH}$ 6.7. The stock solution of $\mathrm{FU}$ was prepared by dissolving it in the appropriate amount of redistilled water. Extra pure nitrogen was used for purging the electrolyte solutions for $10 \mathrm{~min}$ prior to each measurement. A nitrogen blanket over the electrolyte solution was maintained thereafter.

The voltammograms were recorded using an Autolab multi-mode polarograph (ECO Chemie, Utrecht, Netherlands) and PAR 384B polarographic analyzer, which were both connected to a personal computer and a static mercury drop electrode (SMDE), Model 303A from Princeton Applied Research. A platinum wire was used as an auxiliary electrode and $\mathrm{Ag}|\mathrm{AgCl}| \mathrm{KCl}_{\text {sat }}$ as the reference electrode. The measurements were carried out at room temperature of about $22^{\circ} \mathrm{C}$.

\section{Results and discussion}

Square-wave voltammograms of a $5 \times 10^{-5} \mathrm{~mol}^{-1}$ FU solution recorded in 0.1 mol $1^{-1} \mathrm{Na}_{2} \mathrm{SO}_{4}$ in a cathodic stripping regime consist of a single peak with a peak potential of about $0.05 \mathrm{~V}$. Under anodic polarization of the HMD electrode $\left(E_{\text {acc }} \geq 0.1 \mathrm{~V}\right)$ a sparingly soluble compound is formed between FU and mercury, which is deposited on the electrode surface. Under a negative potential scan, the thin film of the insoluble compound is stripped from the electrode surface yielding a cathodic stripping square-wave voltammetric (CSSWV) response. The voltammetric response of this process depends mainly on the $\mathrm{pH}$ of the supporting electrolyte, the potential and time of the accumulation, the FU concentration, as well as the parameters of the exciting SW signal. It has been observed that the cathodic stripping response can be obtained within the $\mathrm{pH}$ region $6-8$. The voltammetric response is best shaped in neutral medium, which is in agreement with the findings of other investigators [6,9]. In acidic medium the CSSWV response was not observed. In basic medium, the peak associated with the intensive dissolution of the mercury overlaps the CSSWV response of FU.

The CSSWV peak of FU is appreciably dependent on both time and accumulation potential. Changing the accumulation potential from 0.15 to $0.0 \mathrm{~V}$ caused the height of the CSSWV response to decrease. The peak current depends on the accumulation potential in the 
form of a pseudopolarogram. If the accumulation potential was more negative than $0.05 \mathrm{~V}$, the peak virtually vanished. It is important to note that under certain experimental conditions that correspond to either high FU concentration or long accumulation time, the CSSWV response of FU consists of two peaks. This phenomenon is attributed to the existence of either a multi-layer deposited film on the electrode surface, or the creation of two complexes with different stoichiometry [10]. A similar phenomenon was observed in the cathodic stripping voltammetry of cysteine and other cathodic stripping active compounds [11-13]. This phenomenon frequently accompanies the cathodic stripping methods, and hence appears to be their main limitation.

In order to get an insight into both formation and dissolution of the insoluble salt formed between $\mathrm{Hg}$ and FU, cyclic staircase voltammetry was employed. Cyclic voltammograms of a $2 \times 10^{-7}$ mol $1^{-1}$ FU solution were recorded without accumulation time and with accumulation time of $20 \mathrm{~s}$ in $0.1 \mathrm{~mol}^{-1} \mathrm{Na}_{2} \mathrm{SO}_{4}$. The deposition of the insoluble salt was carried out at a potential of $0.15 \mathrm{~V}$. The cyclic voltammograms consisted of a cathodic peak, which illustrates the stripping process of the deposited salt, and an anodic peak representing the anodic dissolution of the mercury electrode, followed by creation of the insoluble complex with FU. Increasing the accumulation time caused both components of the cyclic voltammogram to increase. Moreover, under certain experimental conditions, sub-

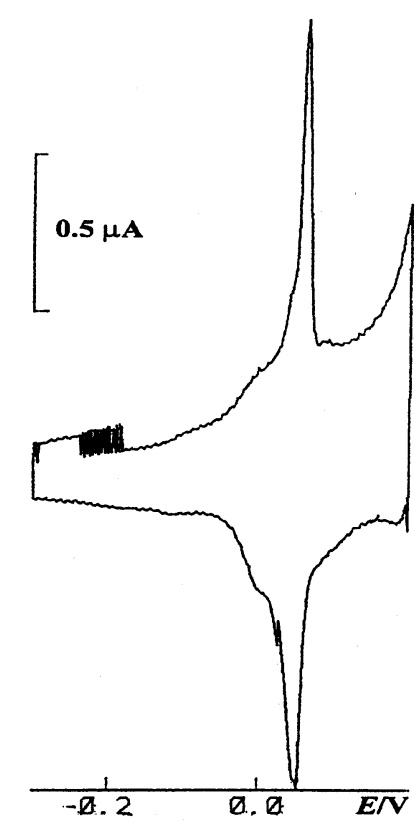

Fig. 1. Cyclic staircase voltammogram of $1 \times 10^{-6} \mathrm{~mol} \mathrm{1}^{-1} \mathrm{FU}+0.1$ mol $1^{-1} \mathrm{Na}_{2} \mathrm{SO}_{4}$ starting from $-0.3 \mathrm{~V}$ towards $0.2 \mathrm{~V}$ and back to $-0.3 \mathrm{~V}$. The accumulation was carried out at $E_{\text {acc }}=-0.3 \mathrm{~V}$ for 15 $\mathrm{s}$. The scan rate was $0.5 \mathrm{~V} \mathrm{~s}^{-1}$. The $\mathrm{pH}$ of the supporting electrolyte was 6.7 . sequent cycling of the potential has no significant influence on either $\mathrm{CV}$ peak, and a steady-state cyclic voltammogram was achieved. These results imply that the redox reaction has the properties of a surface confined redox process in which both the oxidized and the reduced forms of the redox couple are immobilized on the electrode surface. This could be the case only if the FU molecules remain adsorbed on the HMDE surface after stripping of the deposited salt. This assumption is in agreement with the results of Guerrier at al. [10].

At a potential more negative than $0.0 \mathrm{~V}$, the complex with mercury does not exist, and thus within this potential region only the adsorption of FU could occur. In order to support this presumption, the accumulation was carried out at a potential of $-0.3 \mathrm{~V}$, and the SW voltammograms were recorded starting from $-0.3 \mathrm{~V}$ towards the final potential of $0.2 \mathrm{~V}$. Under anodic polarization of the electrode, the SWV response consisted of a sharp and a well defined peak, with a peak potential of $0.052 \mathrm{~V}$. The peak is characterized with a half-peak width of about $100 \mathrm{mV}$, and the overall response is better defined compared with the corresponding peak recorded in the cathodic stripping regime. The SW response recorded under anodic polarization of the electrode is highly sensitive to the accumulation time. The SW peak current depends on the accumulation time in the form of an isotherm, revealing significant adsorption of $\mathrm{FU}$ molecules.

The cyclic voltammogram of a $1 \times 10^{-6} \mathrm{~mol}^{-1} \mathrm{FU}$ solution recorded with an accumulation time of $15 \mathrm{~s}$ at an accumulation potential of $-0.3 \mathrm{~V}$ is presented in Fig. 1. The positive sweep of the cyclic voltammogram (anodic peak) corresponds to the creation of an insoluble salt with mercury and the negative sweep (cathodic peak) appears as a result of the reduction of the salt formed during the previous potential scan. Both peaks are sharply shaped, with an equal peak potential of $0.058 \mathrm{~V}$ and virtually identical height. If the electrode is saturated with adsorbed material (long accumulation time) subsequent cyclizations of the potential had no effect on the response, and hence a steady-state cyclic voltammogram is achieved. Prolonged accumulation time increases proportionally both peaks of the cyclic voltammograms. All these results imply that FU molecules adsorb on the electrode surface, and the formation of the complex with mercury proceeds from an adsorbed state. The overall electrode mechanism could be described by either a first (Eq. (I)) or a second (Eq. (II)) order reaction:

$$
\begin{aligned}
& \mathrm{L}^{2-}(\mathrm{aq}) \leftrightharpoons \mathrm{L}^{2-}(\mathrm{ads})+\mathrm{Hg}(\mathrm{l}) \leftrightharpoons \mathrm{HgL}(\mathrm{s})+2 \mathrm{e}^{-} \\
& 2 \mathrm{~L}^{-}(\mathrm{aq}) \leftrightharpoons 2 \mathrm{~L}^{-}(\mathrm{ads})+2 \mathrm{Hg}(\mathrm{l}) \leftrightharpoons \mathrm{Hg}_{2} \mathrm{~L}_{2}(\mathrm{~s})+2 \mathrm{e}^{-}
\end{aligned}
$$

where $\mathrm{L}^{2-}$ is the symbol for FU as a reacting ligand. In our previous publication [14] we demonstrated that 


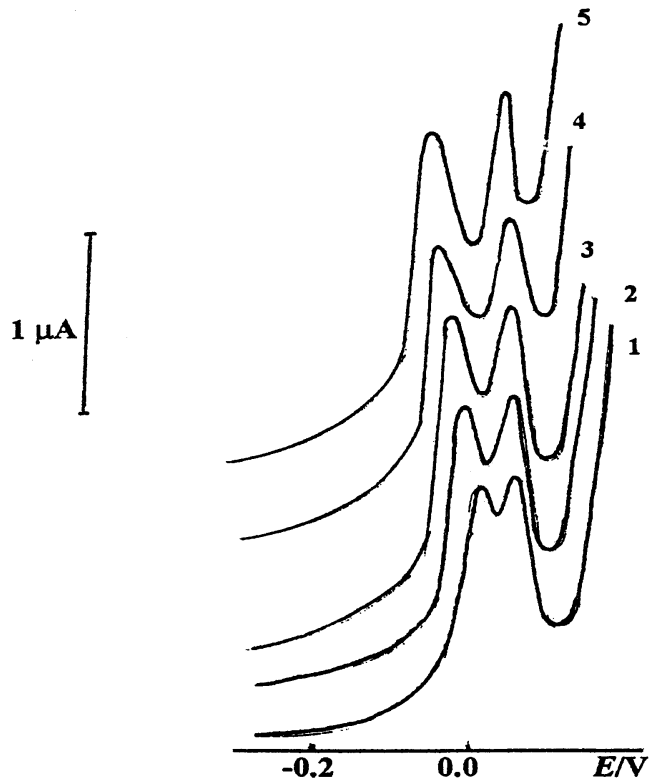

Fig. 2. Splitting of the SWV response of a $1 \times 10^{-6} \mathrm{~mol} 1^{-1} \mathrm{FU}$ solution under increasing amplitude of the signal. The experimental conditions were: the accumulation time $t_{\mathrm{acc}}=15 \mathrm{~s}$, the accumulation potential $E_{\text {acc }}=-0.3 \mathrm{~V}$, the frequency $f=90 \mathrm{~Hz}$, the scan increment $\mathrm{d} E=2 \mathrm{mV}$ and the amplitude $E_{\mathrm{sw}}=45$ (1); 50 (2); 55 (3); 60 (4) and $65 \mathrm{mV}$ (5). All other conditions were the same as in the caption of Fig. 1.

both mechanisms exhibit rather similar voltammetric behavior. However, these redox processes could be distinguished by inspecting the dependence of the peak potential on the concentration of the ligand investigated. If the redox reaction is second order, the peak potential depends linearly on the logarithm of the ligand concentration with a slope of $-2.303 R T /(2 F)$ and of about $-15 \mathrm{mV}$ for reversible and quasi-reversible redox reactions, respectively. The experimental results revealed that there is no significant variation of the peak potential with the concentration of $\mathrm{FU}$ within the concentration interval from $10^{-11}$ to $10^{-7}$ mol $1^{-1}$, indicating that its redox reaction proceeds according to Eq. (I).

The SW voltammetric response of FU depends markedly on the parameters of the exciting signal, such as the frequency $f$ and the amplitude $E_{\mathrm{sw}}$. At a frequency of $90 \mathrm{~Hz}$, the peak height is enhanced in proportion with the amplitude over the range $E_{\mathrm{sw}}=5$ $\mathrm{mV}$ to $E_{\mathrm{sw}}=25 \mathrm{mV}$. The peak potential and the halfpeak width remain virtually the same as the amplitude. If the amplitude was increased up to $E_{\mathrm{sw}}=30 \mathrm{mV}$, the SW peak suddenly split into two peaks. Increasing amplitude caused the potential separation between the split peaks to increase (see Fig. 2). The potential separation $\Delta E_{\mathrm{p}}$ is a linear function of the $\mathrm{SW}$ amplitude with a correlation coefficient of the linear regression line of $R^{2}=0.993$. The ratio of the split peak currents $I_{\mathrm{p} 1} / I_{\mathrm{p} 2}\left(I_{\mathrm{p} 1}\right.$ and $I_{\mathrm{p} 2}$ are the peak currents of the peaks, which appear at more negative and more positive potentials, respectively) does not depend significantly on the SW amplitude and its average value is $0.94 \pm 0.04$.

In order to support the suggested electrode mechanism (I), and moreover, to clarify the complex voltammetric behavior of FU under conditions of SWV, a theoretical model of the proposed electrode mechanism is developed. The mathematical model is given in the Appendix. Theoretical SW voltammograms are compared both qualitatively and quantitatively with the experimental voltammograms and kinetic parameters of the redox reaction of FU at mercury electrode are estimated.

The dimensionless response of the investigated electrode mechanism depends mainly on three dimensionless parameters: $\gamma=\left(1 / r_{\mathrm{s}}\right)(D / f)^{1 / 2}, \rho=K(D / f)^{1 / 2}$, and $\lambda=k_{\mathrm{s}} r_{\mathrm{s}} /(D f)^{1 / 2}$. The parameter $\gamma$ represents the diffusion transport of the reacting ligand towards the electrode surface, $\rho$ reflects the effect of adsorption of the ligand and $\lambda$ appears due to the influence of the charge transfer kinetics.

For a given adsorption constant, the apparent reversibility of the redox reaction (I) depends on a single complex kinetic parameter defined through $\lambda$ and $\gamma$ as: $\omega=\lambda \gamma$. The parameter $\omega$ can be expressed as a function of the real parameters such as the standard rate constant $k_{\mathrm{s}}$ and the frequency of the signal $f: \omega=k_{\mathrm{s}} / f$.

Theoretical investigations confirmed the phenomenon of split SW peaks under increasing signal amplitude. It was observed that the single SW peak splits into two symmetrical peaks if the SW amplitude is increased above a certain critical value. The splitting appears as a consequence of the increased separation between the peak potentials of the forward and backward components of the SW response. Similar behavior was recently observed in the case of surface redox reactions [15-17]. Moreover, this phenomenon was utilized for kinetic measurements of strongly adsorbed redox couples [15]. It should be emphasized that the splitting of the SW peak appears if the surface redox reaction is fast and reversible. However, in the present mechanism, the splitting of the peak is attributed to the quasi-reversible reaction.

The separation between the split peaks $\Delta E_{\mathrm{p}}$ depends mainly on the SW amplitude $E_{\text {sw }}$, the kinetic parameter $\omega$ and the adsorption constant $K$. Numerical simulations revealed that the separation increases proportionally to the SW amplitude. The slope of the lines is approximately the same for all kinetic parameters, however the intercept depends on the particular value of the kinetic parameter.

The potential separation between the split SW peaks of $\mathrm{FU}$ is also a function of the frequency of the SW signal. If the splitting was reached at a certain amplitude, the potential separation decreased with increasing frequency (see Fig. 3). At an amplitude of $40 \mathrm{mV}$, the 
splitting of the peak vanished if the frequency was enlarged above $120 \mathrm{~Hz}$. However, the splitting also disappeared if the frequency was lowered down to 20 $\mathrm{Hz}$, indicating that at a particular amplitude the splitting appeared within a certain frequency interval.

A theoretical study of this phenomenon revealed that for a given amplitude and adsorption constant, the splitting of the SW peak appears only for a strictly defined interval of the kinetic parameter values. For instance, for $E_{\mathrm{sw}}=40 \mathrm{mV}$ and $K=10 \mathrm{~cm}^{-1}$, the splitting of the peak exists only within the interval $-0.5 \leq$ $\log (\omega) \leq 0.1$. For both faster and slower redox reactions, the splitting of the peak vanishes. Within the boundaries of this interval, the separation between the split peaks is a linear function of the kinetic parameter $\omega$. Keeping in mind that the kinetic parameter is defined as $\omega=k_{\mathrm{s}} / f$, these theoretical findings explain the effect of the SW frequency on the split SW peaks of FU (see Fig. 3).

In addition, for given $E_{\mathrm{sw}}$ and $\omega$, the splitting is also associated with a certain interval of the adsorption constant. For instance, for $E_{\mathrm{sw}}=40 \mathrm{mV}$, and $\log (\omega)=$ 0 , the splitting appears within the interval $0.3 \leq$ $\log (K) \leq 1.2$. Inside this interval, the separation is non-linearly dependent on the adsorption constant. The maximum separation is observed for $\log (K)=1.2$. The above results indicate that if the splitting of the SW peak was observed experimentally one could estimate the range of values for both adsorption and kinetic rate constants.

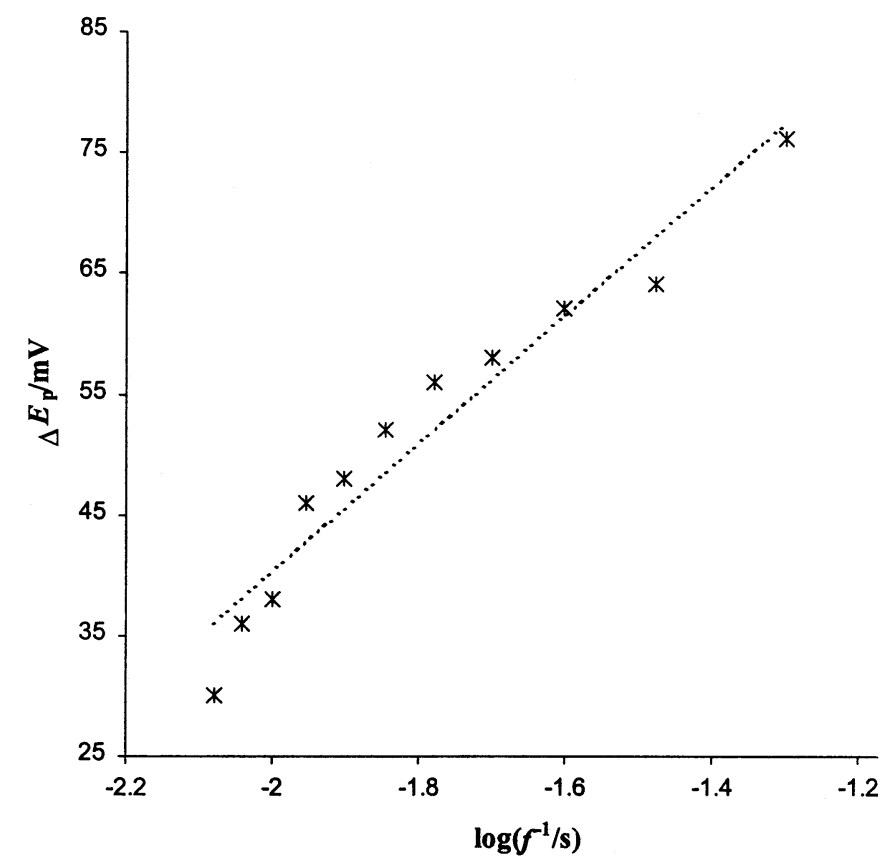

Fig. 3. The dependence of the separation between the peak potentials $\Delta E_{\mathrm{p}}$ of the split $\mathrm{SW}$ response of $\mathrm{FU}$ on the logarithm of the inverse signal frequency. The amplitude was $E_{\mathrm{sw}}=40 \mathrm{mV}$. All other conditions were same as in the caption of Fig. 2.
Numerical simulations showed that the electron transfer coefficient $\alpha$ has a negligible effect on the separation between the split peaks, however, it influences significantly the ratio between peak currents of the split peaks $\Psi_{\mathrm{p} 1} / \Psi_{\mathrm{p} 2}\left(\Psi_{\mathrm{p} 1}\right.$ and $\Psi_{\mathrm{p} 2}$ are the peak currents of the peaks appearing at more negative and positive potentials, respectively). A linear dependence exists between the logarithm of this ratio on the transfer coefficient $\alpha$. The dependence is associated with the following equation: $\log \left(\Psi_{\mathrm{p} 1} / \Psi_{\mathrm{p} 2}\right)=1.3205 \alpha-0.723$. This dependence could be used for the estimation of the cathodic transfer coefficient.

At low SW amplitude, the SW frequency affects the SW peak currents of FU in a different way. A non-linear dependence between the peak currents and the frequency was observed over the frequency range 100 $700 \mathrm{~Hz}$. The ratio between the peak current and the square-root of the corresponding frequency $\Delta I_{\mathrm{p}} / f^{1 / 2}$ depends parabolically on the logarithm of the inverse frequency (see Fig. 4). The curve is characterized by a maximum associated with a critical frequency of $f_{\max }=$ $400 \mathrm{~Hz}$

In order to explain the latest experimental results, the effect of the kinetic parameter $\omega=k_{\mathrm{s}} / f$ on the peak current of the theoretical voltammograms was studied. The dependence of the dimensionless peak current on the kinetic parameter $\omega$ is parabolic. For an adsorption constant $K=10 \mathrm{~cm}^{-1}$, this particular redox reaction seems to be quasi-reversible if $\omega$ ranges within the interval $-1.8 \leq \log (\omega) \leq 0.4$. The quasi-reversible region is marked by a significant enhancement of the dimensionless current. Within the quasi-reversible region, the dimensionless current depends parabolically on the kinetic parameter $\omega$, forming a relatively sharp maximum. This phenomenon is known as a 'quasi-reversible maximum'. It is an essential property of the whole class of electrode processes, which proceed via an adsorbed state of the reactant of the redox reaction [18-23]. The quasi-reversible maximum appears as a consequence of the current sampling procedure used in the SW voltammetry. When the frequency of the signal is synchronized with the charge transfer rate, a multiple and exhaustive re-use of the surface confined material occurs, and thus the SW voltammetry induces a maximal response.

The position of the quasi-reversible maximum is dependent on various factors such as the adsorption constant $K$, the cathodic transfer coefficient $\alpha$ and the SW amplitude $E_{\mathrm{sw}}$. Under the influence of increasing adsorption constant, the quasi-reversible maximum appears at lower values of the parameter $\omega$. Each maximum is associated with a certain critical value of the argument $\omega_{\max }$. A linear relationship exists between the logarithms of the critical argument $\omega_{\max }$ and adsorption constant $K$. The regression line of this dependence is associated by the following equation: $\log \left(\omega_{\max }\right)=$ 


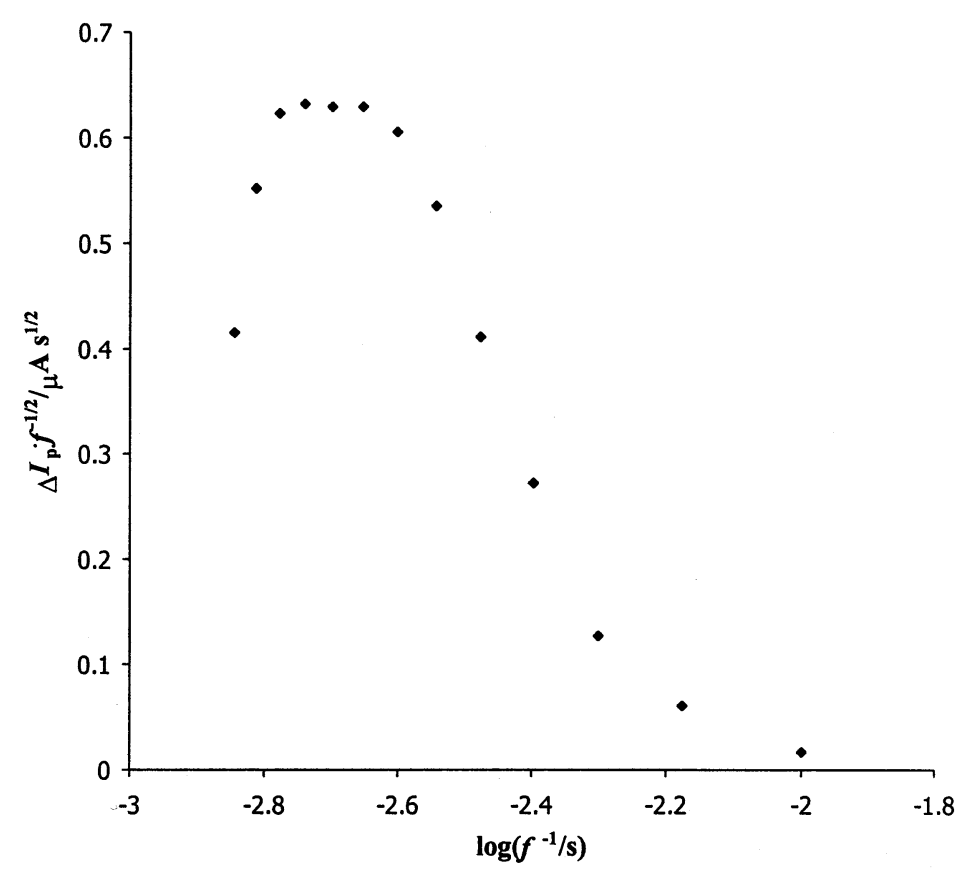

Fig. 4. The dependence of the ratio $\Delta I_{\mathrm{p}} / f^{1 / 2}$ on the logarithm of the inverse signal frequency $f^{-1}$. The amplitude of the signal was $E_{\mathrm{sw}}=25 \mathrm{mV}$. All other conditions were same as in the caption of Fig. 2.

$-0.0042 K-0.5711$ with a correlation coefficient $R^{2}=$ 0.997 .

Investigating experimentally a particular redox reaction, the kinetic parameter $\omega=k_{\mathrm{s}} / f$ could be varied by adjusting the frequency of the signal. Plotting the ratio $\Delta I_{\mathrm{p}} / f^{1 / 2}$ versus $1 / f$ one obtains a dependence, which corresponds to the theoretical dependence of the dimensionless peak current $\Delta \Psi_{\mathrm{p}}$ on the kinetic parameter $\omega$. Therefore, if the critical frequency associated with the quasi-reversible maximum was determined experimentally, and the critical value of $\omega_{\max }$ calculated theoretically, the standard rate constant could be estimated through the following simple relation: $k_{\mathrm{s}}=\omega_{\max } f_{\max }$. A similar procedure was applied in the case of a simple surface redox process of a strongly adsorbed redox couple [22]. Therefore, the above theoretical consideration clarifies the parabolic dependence of the ratio $\Delta I_{\mathrm{p}} / f^{1 / 2}$ on the inverse frequency for $\mathrm{FU}$, presented in Fig. 4.

As we have shown in our previous publications $[15,22,24,25]$, the phenomena of 'split SW peaks' and a 'quasi-reversible maximum' are identifiable features for estimating the electron transfer coefficient $\alpha$ and the standard rate constant $k_{\mathrm{s}}$ of the redox reaction investigated.

It was mentioned previously that the average ratio of the split peaks current $I_{\mathrm{p} 1} / I_{\mathrm{p} 2}$ for $\mathrm{FU}$ is $0.94 \pm 0.04$. Utilizing the equation $\log \left(\Psi_{\mathrm{p} 1} / \Psi_{\mathrm{p} 2}\right)=1.3205 \alpha-0.723$ one can estimate the transfer coefficient $\alpha=0.53$ with a precision of \pm 0.01 . In addition, Fig. 5 shows a comparison between the potential separation of the split peaks $\Delta E_{\mathrm{p}}$ for $\mathrm{FU}$ with the theoretical data simulated under corresponding conditions. The best fit was obtained for a standard rate constant $k_{\mathrm{s}}=54 \mathrm{~s}^{-1}$ and an adsorption constant $K=10 \mathrm{~cm}^{-1}$. A correlation diagram between the experimental and theoretical data is characterized with a correlation coefficient of $R^{2}=$ 0.9992 , which can be regarded as a quantitative indicator for the precision of the applied method.

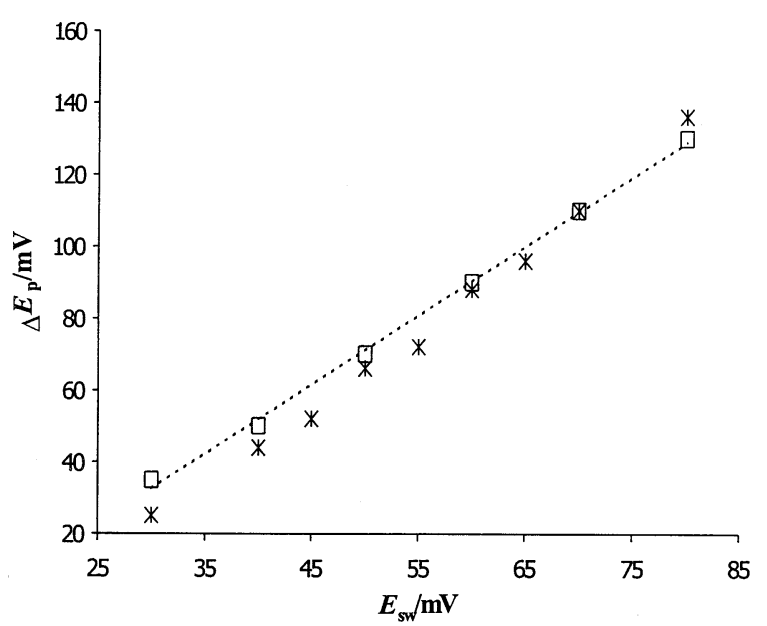

Fig. 5. A comparison of the experimental (asterisks) and the theoretical (squares) data for the separation between the peak potentials $\Delta E_{\mathrm{p}}$ of the split response under the influence of the amplitude. The experimental conditions for FU are same as in the caption of Fig. 2(A). The theoretical data are calculated for the standard rate constant $k_{\mathrm{s}}=54 \mathrm{~s}^{-1}$, the electron transfer coefficient $\alpha=0.5$, and the adsorption constant $K=10 \mathrm{~cm}^{-1}$. The inset shows a correlation diagram between the theoretical and experimental data. 


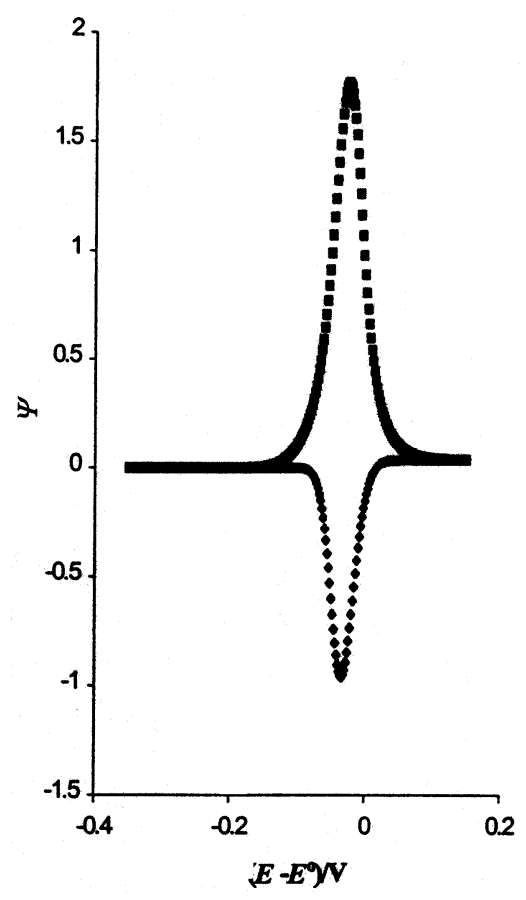

Fig. 6. The theoretical cyclic staircase voltammogram of the redox mechanism (I) simulated under the following conditions: standard rate constant $k_{\mathrm{s}}=15 \mathrm{~s}^{-1}$, adsorption constant $K=10 \mathrm{~cm}^{-1}$, electron transfer coefficient $\alpha=0.5$, starting potential $E_{\mathrm{s}}=-0.35 \mathrm{~V}$, final potential $E_{\mathrm{f}}=0.15 \mathrm{~V}$, accumulation potential $E_{\text {acc }}=-0.35 \mathrm{~V}$, accumulation time $t_{\text {acc }}=1 \mathrm{~s}$, scan increment $\mathrm{d} E=2 \mathrm{mV}$, duration of the potential step $\tau=0.004 \mathrm{~s}$ and diffusion coefficient of the reacting ligand $D=5 \times 10^{-6} \mathrm{~cm}^{2} \mathrm{~s}^{-1}$. Each potential is referred to the standard redox potential of the electrode mechanism (I).

The standard rate constant can also be estimated using the property of the quasi-reversible maximum. The critical frequency associated with the quasi-reversible maximum of FU presented in Fig. 4 is $f_{\max }=$ $400 \mathrm{~Hz}$. For $\alpha=0.53, E_{\mathrm{sw}}=25 \mathrm{mV}$ and $K=10 \mathrm{~cm}^{-1}$, the critical value of the kinetic parameter is $\omega_{\max }=$ 0.24. Calculating the standard rate constant through the equation $k_{\mathrm{s}}=\omega_{\max } f_{\max }$, one obtains $k_{\mathrm{s}}=96 \pm 12$ $\mathrm{s}^{-1}$, which is in close agreement with the previously calculated value.

In order to confirm the validity of the results obtained by SWV, the proposed electrode mechanism was also studied theoretically under conditions of cyclic staircase voltammetry (CSV). The theoretical CSV response depends mainly on the particular values of the adsorption constant $K$, standard rate constant $k_{\mathrm{s}}$ and the duration of the potential step $\tau$. The reversibility of the redox reaction is controlled by the kinetic parameter defined as $\kappa=k_{\mathrm{s}} \tau$. Convenient features, which can serve for characterization of each cyclic voltamogram, are the separation between the potentials of the cathodic and anodic peak $\Delta E_{\mathrm{p}}=E_{\mathrm{p}, \mathrm{c}}-E_{\mathrm{p}, \mathrm{a}}$, as well as the ratio between the peak currents $r=I_{\mathrm{p}, \mathrm{c}} / I_{\mathrm{p}, \mathrm{a}}$. The parameters $\Delta E_{\mathrm{p}}$ and $r$ are highly sensitive to both the adsorption constant $K$ and the kinetic parameter $\kappa$. The typical CSV response of FU, which is presented in Fig. 1 , is characterized with $\Delta E_{\mathrm{p}}=20 \mathrm{mV}$ and $r=0.77$. In order to obtain a corresponding voltammogram with numerical simulations, a large number of theoretical voltammograms were calculated for a variety of values for the standard rate constant $k_{\mathrm{s}}$ and adsorption constant $K$. During these simulations all other parameters were the same as those used in the experimental measurements. The theoretical voltammogram plotted in Fig. 6 is simulated for $k_{\mathrm{s}}=15 \mathrm{~s}^{-1}$ and $K=10 \mathrm{~cm}^{-1}$. It is characterized with the same values of $\Delta E_{\mathrm{p}}$ and $r$ as the experimental voltammogram of FU presented in Fig. 1.

The proposed electrode mechanism can also be studied by inspecting the dependence of the peak potentials and peak currents of both cathodic and anodic peaks on the kinetic parameter $\kappa$. The peak currents of both peaks depend linearly on the kinetic parameter $\kappa$, as is expected for a surface redox process. Within the quasireversible region, this dependence deviates from linearity, which is a consequence of the phenomenon of the quasi-reversible maximum. However, this type of analysis cannot be utilized for estimation of any kinetic parameter, and it can serve only for qualitative characterization of the electrode mechanism. The standard rate constant can be estimated by analyzing the peak potentials of both cathodic and anodic peaks as a function of the kinetic parameter $\kappa$. In the real experiment, this latest parameter can be varied by adjusting the duration of the potential step $\tau$. Fig. 7 represents the dependence of both cathodic and anodic peaks of the CS voltammograms of FU on the duration of the potential step $\tau$. A part of both curves can be approximated with a linear function. The two lines cross at a certain critical value of the duration of the potential step $\tau_{\mathrm{c}}=0.015 \mathrm{~s}$. The same dependence was obtained by analyzing the peak potentials of the theoretically calculated voltammograms as a function of the kinetic parameter $\kappa=k_{\mathrm{s}} \tau$. The critical value of the kinetic parameter $\kappa_{\mathrm{c}}$, at which the lines cross, depends on the particular value of the adsorption constant $K$ and the transfer coefficient $\alpha$. For $K=10 \mathrm{~cm}^{-1}$ and $\alpha=0.5$, the critical value is $\kappa_{\mathrm{c}}=0.15$. Therefore, the standard rate constant can be calculated through the simple relation $k_{\mathrm{s}}=\kappa_{\mathrm{c}} / \tau_{\mathrm{c}}$, and in the case of FU, $k_{\mathrm{s}}=10 \mathrm{~s}^{-1}$. A similar approach for estimation of the standard rate constant in the case of simple surface redox reactions was used by Laviron [27]. All these results are in acceptable agreement with those obtained by SWV.

From an analytical point of view it is particularly important to investigate the voltammetric behavior of $\mathrm{FU}$ in the presence of $\mathrm{Cu}(\mathrm{II})$ ions. Within some boundaries, the $\mathrm{Cu}$ (II) ions significantly enhance the SW peak current of FU, while the peak potential and half-peak width remain virtually the same. For instance, in the presence of $8 \times 10^{-7} \mathrm{~mol}^{-1} \mathrm{Cu}$ (II) ions, 


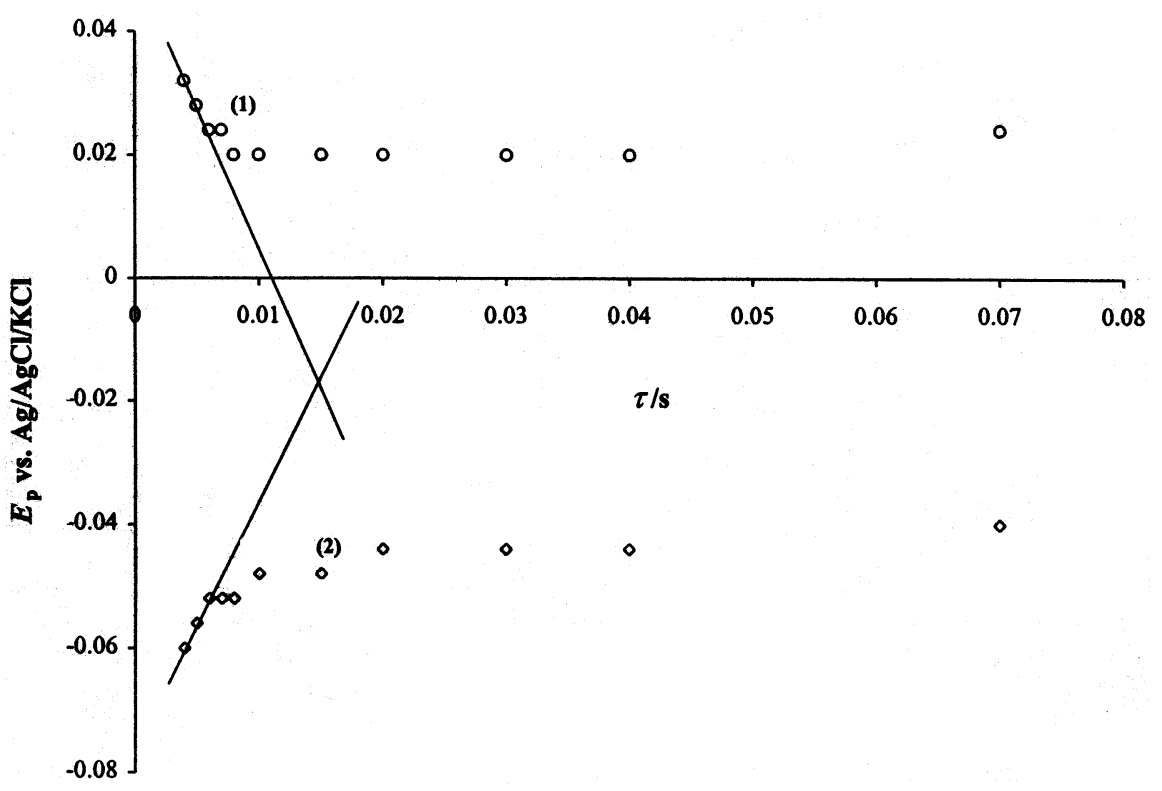

Fig. 7. The dependence of the peak potential of the anodic (1) and cathodic (2) peak of the cyclic staircase voltammograms of a $5 \times 10^{-6}$ mol $1^{-1} \mathrm{FU}$ solution on the duration of the potential step. The voltammograms were recorded in $0.1 \mathrm{~mol}^{-1} \mathrm{Na}_{2} \mathrm{SO}_{4}$ with $\mathrm{pH}$ 6.7. The other experimental conditions were: scan increment $\mathrm{d} E=4 \mathrm{mV}$, accumulation time $t_{\text {acc }}=1 \mathrm{~s}$ and accumulation potential $E_{\text {acc }}=-0.3 \mathrm{~V}$.

the peak of $5 \times 10^{-6} \mathrm{~mol} 1^{-1} \mathrm{FU}$ increases in height by about $50 \%$. The peak current of $5 \times 10^{-6} \mathrm{~mol}^{-1} \mathrm{FU}$ increases in proportion to the concentration of $\mathrm{Cu}(\mathrm{II})$ ions over the range $1-5 \times 10^{-8} \mathrm{~mol} \mathrm{1}^{-1}$. At about a $\mathrm{Cu}(\mathrm{II})$ concentration of $5 \times 10^{-8} \mathrm{~mol} \mathrm{1}^{-1}$, the response reaches a maximum value. Further increase of the $\mathrm{Cu}(\mathrm{II})$ ions exhibits an opposite effect, and hence the peak current diminishes. The overall dependence is parabolically shaped, as shown in Fig. 8. The effect of the $\mathrm{Cu}(\mathrm{II})$ ions appears as a result of the ability of FU to create a stable complex with $\mathrm{Cu}$ (II) $[9,26]$. Investigating the voltammetric behavior of $\mathrm{Cu}$ (II) in the presence of FU, it was observed that the voltammetric response is significantly enhanced and shifted towards more positive potentials in agreement with the findings of other authors [9].

Investigating the influence of the prolonged accumulation on the SW response of $1 \times 10^{-6} \mathrm{~mol} \mathrm{l}^{-1} \mathrm{FU}$ solution without $\mathrm{Cu}(\mathrm{II})$ and in the presence of $5 \times 10^{-7}$ mol $1^{-1} \mathrm{Cu}$ (II) ions, it was observed that the peak currents depend on the accumulation time in the form of an isotherm in both cases. However, the linear portions of the two isotherms were characterized with a different slope, denoting that the adsorption constant of the adsorbate was changed in the presence of $\mathrm{Cu}(\mathrm{II})$.

In addition, numerous cyclic voltammograms of a $5 \times 10^{-6} \mathrm{~mol}^{-1} \mathrm{FU}$ solution were recorded under increasing accumulation time without $\mathrm{Cu}$ (II) and in the presence of $5 \times 10^{-7} \mathrm{~mol} 1^{-1} \mathrm{Cu}(\mathrm{II})$. In the presence of $\mathrm{Cu}$ (II), both peaks of the cyclic voltammograms were proportionally enhanced. The formal potential of the cyclic voltammogram of $\mathrm{FU}$ was $E_{\mathrm{f}}=0.05 \mathrm{~V}$ and it remained at the same value in the presence of $\mathrm{Cu}(\mathrm{II})$. Moreover, the accumulation time shows an identical effect on the cyclic voltammograms of FU in the pres-

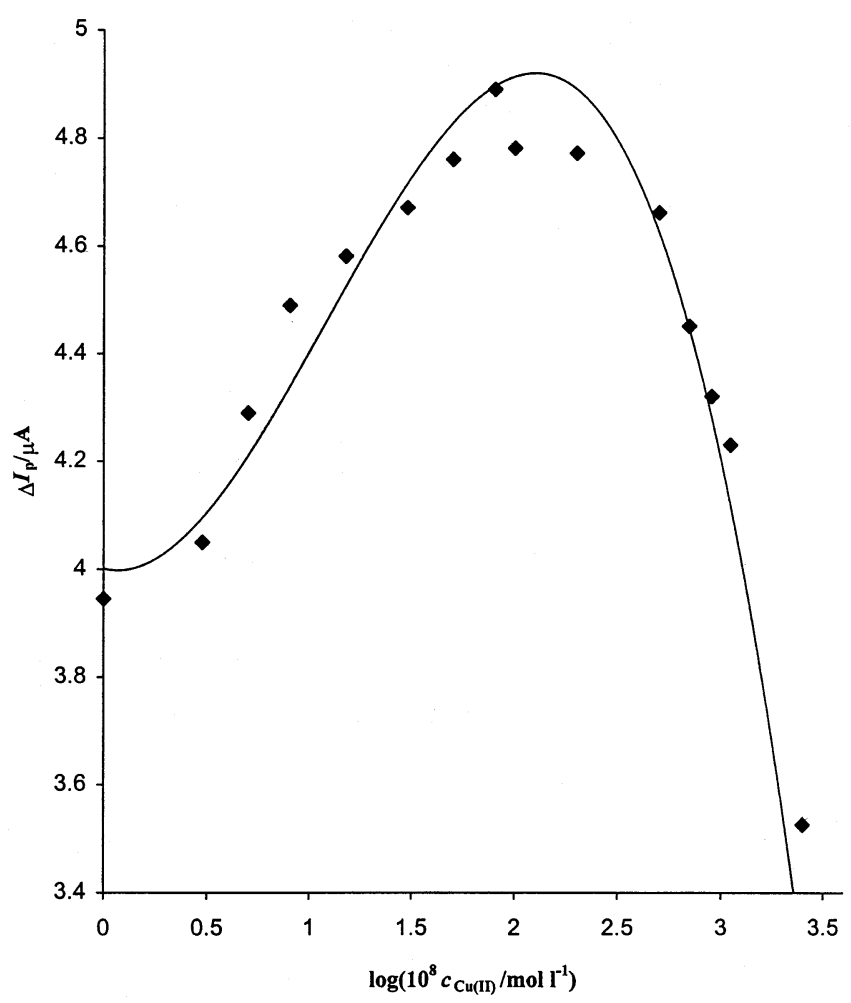

Fig. 8. The dependence of the SW peak currents of the $5 \times 10^{-6} \mathrm{~mol}$ $1^{-1} \mathrm{FU}$ solution on the logarithm of the concentration of $\mathrm{Cu}(\mathrm{II})$ ions. The SW amplitude was $E_{\mathrm{sw}}=20 \mathrm{mV}$, the frequency $f=120 \mathrm{~Hz}$ and the scan increment $\mathrm{d} E=4 \mathrm{mV}$. All other conditions were same as in the captions of Fig. 2(A). 


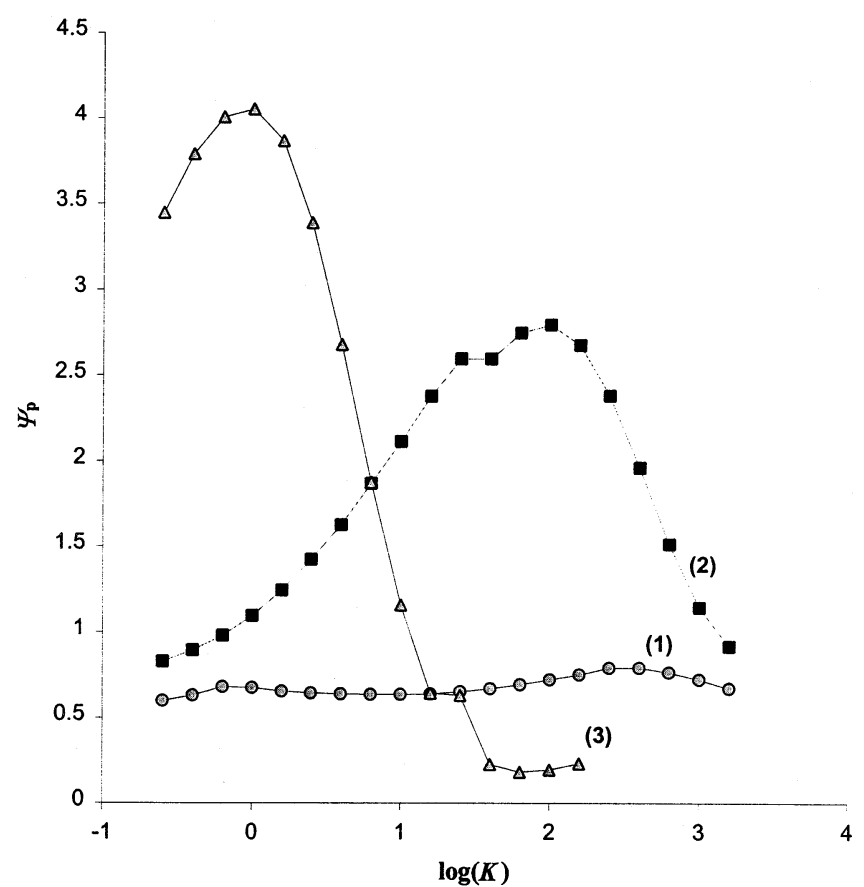

Fig. 9. The dependence of the dimensionless peak currents $\Delta \Psi_{\mathrm{p}}$ on the logarithm of the adsorption constant $K$. The logarithm of the kinetic parameter was $\log (\omega)$ : -2 (1), -1 (2), and 0 (3). The conditions of the simulations were: the accumulation potential $E_{\text {acc }}=$ $-0.2 \mathrm{~V}$ vs. $E^{0}$, the accumulation time $t_{\text {acc }}=1 \mathrm{~s}$, the electron transfer coefficient $\alpha=0.5$, the amplitude $E_{\mathrm{sw}}=20 \mathrm{mV}$, and the scan increment $\mathrm{d} E=5 \mathrm{mV}$.

ence and absence of $\mathrm{Cu}(\mathrm{II})$. All these results clearly imply that the general properties of the redox process of $\mathrm{FU}$ remain the same in the presence of $\mathrm{Cu}(\mathrm{II})$. Nevertheless, it is reasonable to assume that the accumulation of $\mathrm{Cu}-\mathrm{FU}$ complex on the electrode surface is more effective than FU itself, yielding an enhanced voltammetric response of FU. However, so far it is not clear what is the reason for the parabolic dependence of the peak current on the logarithm of the $\mathrm{Cu}$ (II) concentration presented in Fig. 8.

With the aim of explaining the influence of $\mathrm{Cu}(\mathrm{II})$ ions, the dependence of the dimensionless response on the adsorption strength of the reacting ligand was investigated theoretically. If the kinetic parameter $\omega$ is kept at a constant value, the dimensionless response of the mechanism investigated depends on the adsorption strength of the reacting ligand. The dependence of the dimensionless peak current on the adsorption constant is shown in Fig. 9. As can be expected, if the redox reaction behaves irreversibly, the adsorption strength of the reacting ligand has no influence on the dimensionless peak current (Fig. 9, curve 1). However, the effect of the adsorption constant on the dimensionless peak current is significantly pronounced in the quasi-reversible region (Fig. 9, curves 2 and 3). A part of these curves is parabolically shaped with a maximum located around $\log (K)=0$ (for $\log (\omega)=0$ ) and $\log (K)=2$ (for $\log (\omega)=-1)$. This is an important result from an analytical point of view. It reveals that if the reacting ligand is moderately adsorbed on the electrode surface, the current is significantly enhanced, and thus the adsorption phenomenon contributes to the sensitivity of a particular analytical method. Both stronger or weaker adsorption produces an opposite effect.

This behavior appears as a consequence of the specific chronoamperometric properties of this electrode mechanism. The current diminishes exponentially with time during a single potential pulse. Theory revealed that the current values measured after a certain time depend parabolically on the adsorption constant. Therefore, the peak current in SWV, which can be regarded as a complex multi-step chronoamperometric method, depends in the same way on the adsorption constant.

These theoretical results resemble the results obtained for $\mathrm{FU}$ in the presence of $\mathrm{Cu}$ (II) ions (see Fig. 8). From our results, as well as from the literature data $[9,26]$, it is clear that $\mathrm{FU}$ reacts with $\mathrm{Cu}$ (II) yielding a stable complex. The complex formed is adsorbed more effectively at the electrode surface increasing the adsorptive stripping response of FU. However, one should bear in mind that the $\mathrm{Cu}(\mathrm{II})$ ions can also be slightly immobilized on the HMDE surface presumably due to $\mathrm{SO}_{4}^{2-}$ induced adsorption of the $\mathrm{CuSO}_{4}$ complex. Hence, if $\mathrm{Cu}(\mathrm{II})$ ions were present in excess, a concurrent adsorption between $\mathrm{FU}-\mathrm{Cu}(\mathrm{II})$ and $\mathrm{CuSO}_{4}$ complexes occurs. Under such conditions, the adsorption constant of the $\mathrm{Cu}(\mathrm{II})-\mathrm{FU}$ complex changes with concentration of $\mathrm{Cu}(\mathrm{II})$. As a result of this phenomenon a parabolic dependence between $\Delta I_{\mathrm{p}}$ on $\log (\mathrm{Cu}(\mathrm{II}))$ for $\mathrm{FU}$ was observed (see Fig. 8), which is in agreement with the theoretical predictions regarding the peak current-adsorption constant relationship (see Fig. 9).

In the presence of $\mathrm{Cu}(\mathrm{II})$ ions, SWV provides the determination of FU below nano-molar concentration level. The optimal set of experimental conditions for the determination of FU are as follows: accumulation time $t_{\text {acc }}=180 \mathrm{~s}$, accumulation potential $E_{\text {acc }}=-0.3 \mathrm{~V}, \mathrm{SW}$ frequency $f=120 \mathrm{~Hz}$, SW amplitude $E_{\mathrm{sw}}=20 \mathrm{mV}$ and scan increment $\mathrm{d} E=4 \mathrm{mV}$. At $5 \times 10^{-11} \mathrm{~mol}^{1^{-1}}$ $\mathrm{Cu}$ (II) concentration, a linear calibration plot was established within the concentration range $1-9 \times 10^{-11}$ mol $1^{-1}$ with a correlation coefficient of $R^{2}=0.992$. The detection limit of $7.7 \times 10^{-12}$ mol $1^{-1}$ with an accumulation time of $180 \mathrm{~s}$ was estimated according to the formula: $\Delta I_{\min }=\Delta I_{\mathrm{b}}+k S_{\mathrm{b}}$, where $\Delta I_{\mathrm{b}}$ is an average value of the supporting electrolyte currents, $k$ is a confidence level factor (for this calculation $k=3$, which means that the confidence level is $99.6 \%$ ) and $S_{\mathrm{b}}$ is the standard deviation of the current values. The reproducibility of the results in terms of relative standard deviation range from 0.9 to $3.2 \%$ (for ten repetitive measurements). 
The extremely low detection limit of FU attained with square-wave voltammetry is due to both the unique advantages of this technique and the specific properties of the electrode mechanism of FU. From the theoretical results presented above, it is clear that the height of the voltammetric response of the electrode mechanism investigated depends strongly on the reversibility of the redox reaction, reflected through the effect of the kinetic parameter $\omega=k_{\mathrm{s}} / f$, and the strength of adsorption of the reacting ligand presented through the influence of the adsorption constant $K$. Due to the phenomenon of the 'quasi-reversible maximum' (see Fig. 4), and moreover due to the parabolic dependence of the peak current on the adsorption constant (see Fig. 9), the highest voltammetric response could be obtained for a quasi-reversible redox reaction and for a moderate adsorption of the reacting ligand. Adjusting the experiment to satisfy these conditions, the lowest detection limit could be achieved. For all these reasons, it is particularly important to know the magnitudes of the kinetic parameters of the redox reaction, as well as the adsorption constant of the analyte. This conclusion is valid not only for the redox reaction of FU, but for the whole class of redox processes of immobilized redox couples. Finally, it can be concluded that the phenomena of 'split peaks' and 'quasi-reversible maxima', which appear to be unique features of the SWV of an immobilized redox couple, enable estimation of the kinetic parameters by a simple and fast procedure.

Table 1

List of symbols and abbreviations

$\begin{array}{ll}\left(c_{\mathrm{L}}\right)_{\mathrm{s}} & \text { bulk concentration of the ligand ions } \\ c_{\mathrm{L}} & \text { concentration of the ligand ions anywhere in solution } \\ \left(c_{\mathrm{L}}\right)_{0} & \text { concentrations of the } \mathrm{L}^{-} \text {at the electrode surface } \\ D & \text { diffusion coefficient } \\ E & \text { potential of the working electrode } \\ E_{\mathrm{sw}} & \text { SWV pulse height } \\ \mathrm{d} E & \text { SWV scan increment } \\ \Delta E_{\mathrm{p}} & \text { potential separation of the split SWV response } \\ E^{0} & \text { standard potential of reaction (I) } \\ F & \text { Faraday constant } \\ f & \text { frequency } \\ I & \text { current } \\ \Delta I_{\mathrm{p}} & \text { SWV peak current } \\ k_{\mathrm{s}} & \text { standard rate constant } \\ K & \text { adsorption constant } \\ R & \text { gas constant } \\ S & \text { electrode surface area } \\ T & \text { temperature } \\ t & \text { time } \\ \alpha & \text { transfer coefficient } \\ \Gamma_{\mathrm{HgL}} & \text { surface concentration of the insoluble salt } \mathrm{HgL}(\mathrm{s}) \\ \Gamma_{\mathrm{L}} & \text { surface concentration of the adsorbed ligand } \mathrm{L}^{2-} \\ \Psi & \text { dimensionless current } \\ \Delta \Psi_{\mathrm{p}} & \text { dimensionless peak current } \\ & \end{array}$

From an analytical point of view, the possible interference arising from complex sample types should be addressed as the main disadvantage of this approach. In principle, the problem of interference can be addressed by means of separation techniques. These problems will need to be addressed case by case as the specific nature of the problem depends on the type of the sample. Generally speaking, one should expect appreciable interference if some surface active compounds are present in the sample and/or the supporting electrolyte.

\section{Appendix A. Mathematical model}

A theoretical model of the following redox reaction is developed under the conditions of square-wave voltammetry:

$\mathrm{L}^{2-}(\mathrm{aq}) \leftrightharpoons \mathrm{L}^{2-}(\mathrm{ads})+\mathrm{Hg}(\mathrm{l}) \leftrightharpoons \mathrm{HgL}(\mathrm{s})+2 \mathrm{e}^{-}$

The symbol $\mathrm{L}^{2-}$ represents 5 -fluorouracil as a reacting ligand. It is presumed that the reacting ligand $\mathrm{L}^{2-}$ adsorbs at the electrode surface according to a linear adsorption isotherm: $\left(c_{\mathrm{L}}\right)_{0}=K \Gamma_{\mathrm{L}}$, where $K$ is an adsorption equilibrium constant expressed in $\mathrm{cm}^{-1}$. This approximation is valid for a low surface concentration of FU. In addition, it is presumed that no lateral interactions between deposited particles of $\mathrm{HgL}(\mathrm{s})$, as well as adsorbed $\mathrm{L}^{2-}$ (ads) species exist.

For the meaning of all symbols and abbreviations see Table 1. Mass transport is assumed to occur through a planar, stationary and semi-infinite diffusion. This simplification is justified for short accumulation of the ligand $\mathrm{L}^{2-}$ on the surface of the HMD electrode. The diffusion equation:

$\partial c_{\mathrm{L}} / \partial t=D\left(\partial^{2} c_{\mathrm{L}} / \partial x^{2}\right)$

with the following starting and boundary conditions:

$$
\begin{aligned}
& t=0, x \geq 0: c_{\mathrm{L}}=\left(c_{\mathrm{L}}\right)_{\mathrm{s}}, \Gamma_{\mathrm{HgL}}=0, \Gamma_{\mathrm{L}}=0 \\
& t>0, x \rightarrow \propto: c_{\mathrm{L}} \rightarrow\left(c_{\mathrm{L}}\right)_{\mathrm{s}} \\
& x=0:\left(c_{\mathrm{L}}\right)_{0}=K \Gamma_{\mathrm{L}}, D\left(\partial c_{\mathrm{L}} / \partial x\right)_{0}-\mathrm{d} \Gamma_{\mathrm{L}} / \mathrm{d} t=\mathrm{d} \Gamma_{\mathrm{HgL}} / \mathrm{d} t \\
& -I /(2 F S)=D\left(\partial c_{\mathrm{L}} / \partial x\right)_{0}-\mathrm{d} \Gamma_{\mathrm{L}} / \mathrm{d} t \\
& I /(2 F S)=-\mathrm{d} \Gamma_{\mathrm{HgL}} / \mathrm{d} t
\end{aligned}
$$

was solved using Laplace transformations. The initial time $t=0$ is set at the moment when the working electrode is polarized at the accumulation potential. The solutions reads:

$$
\Gamma_{\mathrm{HgL}}=-\int_{0}^{t} \frac{I(t)}{2 F S} \mathrm{~d} t
$$




$$
\begin{aligned}
\left(c_{\mathrm{L}}\right)_{0}= & \left(c_{\mathrm{L}}\right)_{\mathrm{s}}\left(1-\mathrm{e}^{a^{2} t} \operatorname{erfc}(a \sqrt{t})\right) \\
& +a \int_{0}^{t} \frac{I(\tau)}{2 F S \sqrt{D}}\left[\mathrm{e}^{a^{2}(t-\tau)} \operatorname{erfc}(a \sqrt{t-\tau})\right] \mathrm{d} t
\end{aligned}
$$

where $a=K D^{1 / 2}$.

If the oxidation of the mercury electrode and the reduction of the deposited salt are kinetically controlled processes, the condition at the electrode surface reads:

$$
I /(2 F S)=k_{\mathrm{s}} \mathrm{e}^{-\alpha \phi}\left[\Gamma_{\mathrm{HgL}}-\left(c_{\mathrm{L}}\right)_{0} r_{\mathrm{s}} \mathrm{e}^{\phi}\right]
$$

where $k_{\mathrm{s}}$ is a standard rate constant expressed in units of $\mathrm{s}^{-1}$ and $\phi$ is relative dimensionless electrode potential $\phi=2 F\left(E-E^{0}\right) /(R T)$ and $r_{\mathrm{s}}=1 \mathrm{~cm}$ is an auxiliary constant. Combining Eqs. (2)-(4) the following integral equation was obtained:

$$
\begin{aligned}
\frac{I}{2 F S}= & k_{\mathrm{s}} \mathrm{e}^{-\alpha \phi}\left[-\int_{0}^{t} \frac{I(t)}{2 F S} \mathrm{~d} \tau\right. \\
& -r_{\mathrm{s}} \mathrm{e}^{\phi}\left[\left(c_{\mathrm{L}}\right)_{\mathrm{s}}\left(1-\mathrm{e}^{a^{2} t} \operatorname{erfc}(a \sqrt{t})\right)\right. \\
& \left.+a \int_{0}^{t} \frac{I(\tau)}{2 F S \sqrt{D}}\left(\mathrm{e}^{a^{2}(t-\tau)} \operatorname{erfc}(a \sqrt{t-\tau))}) \mathrm{d} \tau\right]\right]
\end{aligned}
$$

The above equation can be regarded as a mathematical solution for the electrode mechanism (I). The solutions for Eq. (A5) under conditions of SW voltammetry were obtained using the numerical method of Nicholson and Olmstead [28]. Both variables, current $I$ and time $t$, were incremented. To each time $t=m d$, where $d$ is a time increment, a certain current $I_{m}$ was ascribed. The solution for a quasi-reversible redox reaction reads: applied to the working electrode. For the numerical simulation a time increment $d=1 /(50 f)$ was used. It means that each SW half-period $\tau / 2$ was divided into 25 increments. The square-wave potential signal is a train of negative and positive pulses superposed onto a staircase potential ramp. The signal is characterized with the amplitude $E_{\mathrm{sw}}$, which is half of the peak to peak height, the frequency of the pulses $f$ and the scan increment $\mathrm{d} E$.

\section{References}

[1] C. Heidlberg, F.J. Ansfield, Cancer Res. 23 (1963) 1266.

[2] R. Boulieu, C. Bory, C. Gonet, J. Chromatogr. 339 (1985) 380.

[3] R.C. Simpson, P.R. Brown, J. Chromatogr. 379 (1986) 311.

[4] O. Manousek, P. Zuman, Chem. Listy 49 (1956) 668.

[5] M. Wrona, B. Czochralska, Acta Biochim. Pol. 17 (1970) 351.

[6] B. Bouzid, A.M.G. Macdonald, Anal. Chim. Acta 211 (1988) 155.

[7] A.J. Miranda, M.J. Garcia Gutierrez, A.C. Garcia, P.T. Blanco, W.F. Smyth, Analyst 112 (1987) 243.

[8] J. Wang, M.S. Lin, V. Villa, Analyst 112 (1987) 247.

[9] M. Khodari, M. Ghandour, A.M. Taha, Talanta 44 (1997) 305.

[10] A. Guerrieri, T.R.I. Cataldi, F. Palmisano, P.G. Zambonin, J. Electroanal. Chem. 314 (1991) 117.

[11] T.M. Florence, J. Electroanal. Chem. 219 (1979) 237.

[12] M.T. Stankovich, A.J. Bard, J. Electroanal. Chem. 75 (1977) 487.

[13] I.R. Miller, J. Teva, Electroanal. Chem. 36 (1972) 157.

[14] V. Mirčeski, M. Lovrić, Electroanalysis 10 (1998) 975.

[15] V. Mirčeski, M. Lovrić, Electroanalysis 9 (1997) 1283.

[16] J.J. O'Dea, A. Ribes, J.G. Osteryoung, Anal. Chem. 65 (1993) 3090.

[17] J.H. Reeves, S. Song, E.F. Bowden, Anal. Chem. 65 (1993) 683.

[18] M. Lovrić, I. Pižeta, Š. Komorsky-Lovrić, Electroanalysis 4 (1992) 327.

[19] M. Lovrić, Š. Komorsky-Lovrić, J. Electroanal. Chem. 248 (1988) 239.

$$
\psi_{m}=\frac{-\frac{\lambda \gamma \mathrm{e}^{-\alpha \phi_{m}}}{50} \sum_{i=1}^{m-1} \Psi_{i}-\lambda \mathrm{e}^{(1-\alpha) \phi_{m}}\left[\left(1-\mathrm{e}^{\left(\frac{\rho^{2} m}{50}\right)}\right)\left(1-\operatorname{erf}\left(\rho \sqrt{\frac{m}{50}}\right)\right)+\frac{1}{\sqrt{50}} \sum_{i=1}^{m-1} \Psi_{i}\left[\frac{2 S_{m-i+1}}{\sqrt{\pi}}+\frac{\sqrt{50}}{\rho} R_{(m-i+1)}\right]\right]}{1+\frac{\lambda \gamma}{50} \mathrm{e}^{-\alpha \phi_{m}}+\frac{\lambda e^{(1-\alpha) \phi_{m}}\left(\frac{2 S_{1}}{\sqrt{\pi}}+\frac{\sqrt{50}}{\rho} R_{1}\right)}{\sqrt{50}}}
$$

where $\Psi$ is a dimensionless current $\Psi=I(D f)^{-1 / 2}$ $\left(2 F S\left(c_{\mathrm{L}}\right) \mathrm{s}, \gamma\right.$ is a diffusion parameter $\gamma=\left(1 / r_{\mathrm{s}}\right)(D / f)^{1 / 2}$, $\rho$ is an adsorption parameter $\rho=K(D / f)^{1 / 2}, \lambda$ is a kinetic parameter $\lambda=k_{\mathrm{s}} r_{\mathrm{s}} /(D f)^{1 / 2}$, and

$R_{m}=\mathrm{e}^{\left(\frac{\rho m}{50}\right)} \operatorname{erfc}\left(\rho \sqrt{\frac{m}{50}}\right)-\mathrm{e}^{\frac{\rho(m-1)}{50}} \operatorname{erfc}\left(\rho \sqrt{\frac{m-1}{50}}\right)$

and

$S_{m}=\sqrt{m}-\sqrt{m-1}$

For $1 \leq m \leq M_{1}$ (where $M_{1}=t_{\mathrm{acc}} \mathrm{d}, t_{\mathrm{acc}}$ is the accumulation time) the electrode is polarized at a constant accumulation potential $E_{\text {acc. }}$. In the period $M_{1} \leq m \leq M$ ( $M=t_{1} d$, where $t_{1}$ is total time calculated as a sum of accumulation time and time of the stripping scan) a square-wave excitation signal with frequency $f$ was
[20] Š. Komorsky-Lovrić, M. Lovrić, Fresenius' Z. Anal. Chem. 335 (1989) 289.

[21] Š. Komorsky-Lovrić, M. Lovrić, J. Electroanal. Chem. 384 (1995) 115.

[22] Š. Komorsky-Lovrić, M. Lovrić, Anal. Chim. Acta 305 (1995) 248.

[23] M. Lovrić, Š. Komorsky-Lovrić, A.M. Bond, J. Electroanal. Chem. 319 (1991) 1.

[24] V. Mirčeski, M. Lovrić, B. Jordanoski, Electroanalysis 11 (1999) 660.

[25] V. Mirčeski, M. Lovrić, Anal. Chim. Acta 386 (1999) 47.

[26] G.D. Christian, W.C. Purdy, Biochim. Biophys. Acta 54 (1961) 587.

[27] E. Laviron, J. Electroanal. Chem. 101 (1979) 19.

[28] R.S. Nicholson, M.L. Olmstead, in: J.S. Mattson, H.B. Mark, H.C. MacDonald (Eds.), Electrochemistry: Calculations, Simulation and Instrumentation, vol. 2, Marcel Dekker, New York, 1972, pp. $120-137$. 